\title{
Análise de intervenção de PEP e PEPRO sobre os preços do milho no estado de Mato Grosso, de 2009 a 2016
}

\author{
PEP and PEPRO intervention analysis on the maize prices in Mato Grosso state, \\ 2009 to 2016
}

\author{
Análisis de intervención de PEP y PEPRO sobre los precios del maíz en el estado \\ de Mato Grosso, de 2009 a 2016 \\ Douglas Paranahyba de Abreu ${ }^{1}$ \\ Alcido Elenor Wander ${ }^{1}$ \\ Marcelo Dias Paes Ferreira ${ }^{1}$
}

Recebido em: 21/02/2018; revisado e aprovado em: 16/05/2018; aceito em 17/06/2018.

DOI: http://dx.doi.org/10.20435/inter.v21i2.1852

\begin{abstract}
Resumo: O Brasil tem políticas específicas direcionadas aos mercados agrícolas, sendo a Política de Garantia de Preços Mínimos uma das principais e mais antigas. Objetiva-se, por meio de Modelos de Intervenção, captar o efeito dos instrumentos Prêmio para Escoamento de Produto (PEP) e Prêmio Equalizador Pago ao Produtor (PEPRO) sobre os preços do milho no estado de Mato Grosso, principal produtor deste grão. Os resultados demonstram que as intervenções via PEP e PEPRO apresentam pouco efeito sobre os preços, de 2009 a 2016.
\end{abstract}

Palavras-chave: política pública; renda agrícola; commodity.

Abstract: Brazil has specific policies directed at agricultural markets, with the Minimum Price Guarantee Policy being one of the most important and oldest. The objective of this article is to capture the effect of the Program for Product Flow (PEP) and the Equalizing Premium Paid to Grower (PEPRO) instruments on maize prices in the state of Mato Grosso using time series and intervention analysis. The results show that PEP and PEPRO interventions have a negligible effect on prices from 2009 to 2016.

Keywords: public policy; agricultural income; commodity.

Resumen: Brasil tiene políticas específicas dirigidas a los mercados agrícolas, y la Política de Garantía de Precios Mínimos es una de las más importantes y antiguas. El objetivo de los modelos de intervención es capturar el efecto de los instrumentos Programa para Flujo de Producto (PEP) y Premio Ecualizador Pagado al Productor (PEPRO) sobre los precios del maíz en el estado de Mato Grosso, el principal productor de este grano. Los resultados muestran que las intervenciones de PEP y PEPRO tienen poco efecto en los precios desde 2009 hasta 2016.

Palabras clave: política pública; renta agrícola, commodity.

\section{INTRODUÇÃO}

Para tentar assegurar a estabilidade da oferta e da renda do produtor rural, diversos países lançam mão de instrumentos de políticas agrícolas. No Brasil, algumas políticas públicas são aplicadas sobre os mercados agrícolas, principalmente as subordinadas à Política Agrícola. A Política Agrícola brasileira é formada pelo tripé: Política de Garantia de Preços Mínimos (PGPM), Política de Crédito Rural e Política de Seguro Rural (BRASIL, 2016). Entre essas, a PGPM procura exercer influência mais direta sobre os preços dos produtos agrícolas.

Entre os produtos agrícolas brasileiros, o milho é um dos principais pela sua ampla utilização na alimentação animal, principalmente de aves e suínos. Por suas características técnicas de plantio, pode ser facilmente comutado pela soja, conferindo a este produto um importante diferencial para ofertantes e demandantes, principalmente no mercado interno brasileiro

\footnotetext{
${ }^{1}$ Universidade Federal de Goiás (UFG), Goiânia, Goiás, Brasil.
} 
(CALDARELLI; BACCHI, 2012). Mato Grosso apresenta alta representatividade na produção de milho. Nos anos de 2013, 2014, 2015 e 2016, o estado foi o maior produtor de milho do país, responsável por aproximadamente $25 \%$ da produção nacional (IBGE, 2017). Segundo Bacha e Caldarelli (2008), os novos instrumentos de PGPM apresentam concentração espacial e por produto, ganhando destaque na concentração geográfica o estado de Mato Grosso e, na concentração por produto, o milho e a soja.

O Brasil, apesar de possuir um Estado historicamente intervencionista em diversos campos da economia, não apresenta forte característica na avaliação das políticas públicas. Segundo Borges et al. (2011), só a partir da década de 1990 é que houve uma preocupação maior relativa a essas questões, em que a gestão das ações do Estado começou a ser vista como instrumento importante para estabilização da economia. Já com relação a políticas que visam apoiar a comercialização agrícola, Winters, Maffioli e Salazar (2011) argumentam que existe uma carência de documentos que avaliem a sua eficácia, característica essa mais presente nos países em desenvolvimento. O Instituto de Pesquisa Econômica Aplicada (IPEA) do Brasil vem empregando esforços no sentido de contornar a carência de estudos que realizem análise de impacto das ações do Governo. Essa tendência pode ser percebida em Resende (2014a; 2014b; 2017), em que modelos econométricos são propostos e testados para essas finalidades.

Desta forma, percebe-se uma necessidade de avaliação das intervenções do Governo na economia do Brasil. E, quando se tem uma política específica, como é o caso da Política Agrícola, que atua em um setor de grande relevância para o país, esforços no sentido de avaliar o desempenho de sua atuação se fazem importantes. Principalmente para apresentar à sociedade a contrapartida das intervenções e de fomentar a tomada de decisão de formuladores de políticas. Como a Política de Garantia de Preços Mínimos realiza intervenções diretamente nos preços agrícolas, sendo Mato Grosso o principal produtor de milho do país, esse recorte se justifica quando se pretende avaliar intervenções dos instrumentos de PGPM sobre a série de preços do milho.

Com Saraiva et al. (2014) demonstrando que modelos de intervenção são adequados para realizar esse tipo de análise, o objetivo geral deste artigo é realizar análise de intervenção sobre a série de preços de milho no estado de Mato Grosso, tendo como marco temporal da intervenção a data de divulgação das Políticas de Preços-Subsídio da PGPM, que são: Prêmio para Escoamento de Produto (PEP) e Prêmio Equalizador Pago ao Produtor (PEPRO). A hipótese é de que, apesar da grande quantidade de variáveis que influenciam as variações dos preços do milho em Mato Grosso, ao se acionar os instrumentos da PGPM, os preços de mercado reagem positiva e significativamente a esses dispositivos de intervenção.

Assim como apresentam Abreu, Wander e Ferreira (2017), existem no Brasil, desde 2000, cerca de 20 artigos publicados em periódicos científicos que abordam o tema PGPM. Sendo uma das principais políticas públicas ligadas à renda agrícola, é tímida a atuação da academia em discutir esse tipo de intervenção governamental. Além de poucos estudos que abordam a PGPM no Brasil, não é possível perceber um consenso entre os autores em qual seria a metodologia mais adequada para essa temática (ABREU; WANDER; FERREIRA, 2017). No entanto Saraiva et al. (2014) realizam uma análise de intervenção dos instrumentos de PGPM sobre séries de preços agrícolas. A partir desse modelo, é possível verificar se a ação do Governo foi capaz de elevar os preços de mercado, principal variável determinante da renda agrícola. Tal abordagem trata de uma metodologia elaborada por Box e Tiao (1975) adequada à classe de modelos propostos por Box e Jenkins (1976). 
A metodologia de análise de intervenção tem sido utilizada em um campo vasto de investigações: para medir impactos de eventos externos sobre exportações, tal como Ferreira et al. (2011); para identificar efeitos de atentados terroristas sobre taxas de transtorno, em Hansen et al. (2016); e para medir os efeitos de eventos climáticos e econômicos sobre exportações, em Sousa et al. (2010). O presente trabalho não busca esgotar as discussões acerca das formas de se avaliar os efeitos da PGPM sobre a renda agrícola, tendo em vista que tal comparação entre metodologias recairia sobre o campo da pesquisa em epistemologia. No entanto é possível observar em Borgatto e Sáfadi (2000) que houve uma melhoria em seus modelos ao utilizarem efeitos de intervenção. Os autores puderam inserir em suas séries informações decorrentes de políticas públicas sobre algumas variáveis referentes ao transporte coletivo em São Paulo (número médio de passageiro por ônibus, número de assaltos no ônibus e o número de acidentes com os ônibus).

Já Chain et al. (2016) utilizaram modelos de intervenção para medir os efeitos de medidas protecionistas sobre a distância econômica entre Brasil e Argentina, ou seja, de que forma a intervenção governamental interferiu nos fluxos comerciais entre esses dois países. Os autores verificaram que, além de medidas protecionistas, crises econômicas também surtiram efeitos sobre a distância comercial entre os dois países. Dessa maneira, é possível perceber na literatura que, além de auxiliar na modelagem de séries temporais, a análise de intervenção auxilia na identificação de fenômenos, tais como políticas públicas sobre variáveis econômicas. Esse ferramental vem se mostrando capaz de avaliar a influência de determinados efeitos sobre séries de interesse.

Além desta introdução, o artigo é composto de Revisão de Literatura, que buscará apresentar as origens e principais mudanças dessa política, bem como apresentar outros trabalhos que utilizaram o modelo proposto; na seção Metodologia, será apresentada a forma como os dados foram levantados e como os modelos foram estimados; em Resultados e Discussão, serão apresentados os resultados dos modelos estimados, bem como uma breve discussão; e, nas Considerações Finais, uma breve consideração das implicações de tais resultados.

\section{REVISÃO DE LITERATURA}

\subsection{Política de Garantia de Preços Mínimos}

A Política Agrícola brasileira, especificamente a de política de preços mínimos, apresentou grandes mudanças ao longo das últimas décadas. A partir da década de 1990, devido a excessos de estoques combinados com restrição fiscal do Governo Federal e a estabilização macroeconômica, instrumentos tradicionais foram substituídos pelos chamados "novos instrumentos", que diminuíam a quantidade de recursos necessários para manter os preços mínimos nos mercados agrícolas (REZENDE, 2000).

A PGPM sofreu diversas alterações, principalmente em relação aos seus instrumentos tradicionais, quais sejam: Aquisições do Governo Federal (AGF) e os Empréstimos do Governo Federal (EGF). Para Ramos e Morceli (2010), tais mudanças ocorreram no sentido de desonerar o Governo Federal dos altos gastos com a Política de Garantia de Preços Mínimos, principalmente com a estratégia de inserir agentes privados nas operações. No Quadro 1, são apresentadas brevemente as definições dos principais instrumentos de PGPM utilizados atualmente. 
Quadro 1 - Resumo dos principais instrumentos da PGPM

\begin{tabular}{|c|c|}
\hline Nome & Definição \\
\hline $\begin{array}{c}\text { Aquisição do Governo } \\
\text { Federal (AGF) }\end{array}$ & $\begin{array}{c}\text { Composição do estoque público pelo Governo com compra diretamente do } \\
\text { produtor que armazena o produto em locais cadastrados pela Conab. }\end{array}$ \\
\hline $\begin{array}{c}\text { Contrato de Opção } \\
\text { de Venda de Produto } \\
\text { Agrícola (COVPA) }\end{array}$ & $\begin{array}{c}\text { Título ofertado em leilão pelo Governo para produtores e cooperativas. O } \\
\text { arrematante tem a opção, na data de vencimento do contrato, de vender seu } \\
\text { produto para compor estoque público pelo preço fixado na emissão do título. }\end{array}$ \\
\hline $\begin{array}{c}\text { Prêmio de Risco de } \\
\text { Opção Privada (PROP) }\end{array}$ & $\begin{array}{c}\text { Semelhante ao COV, só que o setor privado é quem lança o contrato. } \\
\text { O Governo paga ao agente privado um prêmio (estabelecido em leilão) } \\
\text { referente à diferença entre o preço mínimo e o preço de mercado na data de } \\
\text { vencimento do contrato. }\end{array}$ \\
\hline $\begin{array}{c}\text { Prêmio e Valor de } \\
\text { Escoamento do }\end{array}$ & $\begin{array}{c}\text { Prêmio pago pelo Governo referente à diferença entre o preço mínimo e } \\
\text { o preço de mercado (geralmente equivalente ao frete entre duas regiões), } \\
\text { utilizado para transferir produtos entre duas regiões. O VEP é para estoques } \\
\text { públicos. }\end{array}$ \\
\hline $\begin{array}{c}\text { Prêmio Equalizador } \\
\text { Pago ao Produtor } \\
\text { (PEPRO) }\end{array}$ & $\begin{array}{c}\text { Prêmio pago ao produtor (que participa do leilão) referente à diferença entre } \\
\text { o preço mínimo (ou outro preço estabelecido) e o preço de mercado. }\end{array}$ \\
\hline
\end{tabular}

Fonte: Adaptado de Brasil (2016, p. 30-3).

A partir do Quadro 1, assim como caracterizado por Schwantes e Bacha (2015), é possível distinguir estes instrumentos de PGPM em três tipos de política: Política de Compra de Excedentes pelo Governo (característica do AGF); Política de Seguro de Preços (Característica do COVPA e PROP); e Política Preço-Subsídio (característica do PEP e PEPRO).

\subsection{Abordagens Metodológicas}

Apesar da metodologia de análise de intervenção se mostrar propícia para este tipo de estudo, outras metodologias também são utilizadas para estudar a PGPM. Um exemplo é Bitencourt; Barczsz e Andrade (2008), que avaliaram a PGPM como instrumento de intervenção do Governo no mercado brasileiro de algodão em pluma, milho e trigo, utilizando como metodologia os somatórios de vendas e estoques desses produtos, no intuito de encontrar o estoque máximo realizado pelo Governo. Assim, os autores puderam identificar mudanças importantes que vêm ocorrendo na PGPM ao longo dos anos, tais como a maior participação de agentes privados nas operações de garantia de preços mínimos.

Efeitos positivos dos instrumentos de PGPM sobre as séries de preços agrícolas foram encontrados por Conceição (2002), Pereira; Carvalho e Conceição (2005) e Cruz e Teixeira (2006). Por meio de uma metodologia de cálculo do coeficiente de variação e decomposição da variância, após a institucionalização de alguns instrumentos da PGPM para determinados mercados no Brasil (arroz, milho, algodão e trigo), tais trabalhos verificaram uma redução do risco dessas atividades, percebida pela redução do nível de variação dos preços. No entanto os próprios autores apontam para possíveis interferências de variáveis macroeconômicas sobre seus resultados, tais como a abertura comercial e o Plano Real.

Para Pereira e Vieira (2009), o PEP, que é realizado via leilão, apresenta características de ineficiência econômica. Os autores puderam chegar a essa conclusão utilizando como metodologia a Teoria dos Leilões e sugerem que exista uma quantidade maior de participantes para que o Governo não tenha sempre de pagar o prêmio máximo ao beneficiário. 
Del Bel Filho e Bacha (2005), Bacha e Caldarelli (2008), Osaki e Batalha (2009), Ramos e Morceli (2010) e Massuquetti, Souza e Beroldt (2010) avaliaram os efeitos de instrumentos da PGPM utilizando análise tabular e gráfica de dados secundários. Esses trabalhos apontam pontos positivos e negativos da intervenção governamental via PGPM, ora influenciando positivamente a renda dos produtores, ora causando externalidades negativas com efeitos inócuos sobre a renda dos produtores. Sanches e Bacha (2015) também buscaram identificar os efeitos da PGPM, porém esse trabalho é concluído com uma sugestão que se estende aos demais que buscaram analisar o efeito da PGPM sobre os preços de mercado; ademais, reforça que tais análises devem vir acompanhadas de modelos econométricos capazes de identificar de forma mais isolada os efeitos da intervenção governamental via PGPM sobre os mercados agrícolas.

\section{METODOLOGIA}

Dois tipos de dados são necessários para cumprir com os objetivos desta investigação. O primeiro são as séries de preços do milho no estado de Mato Grosso. O segundo são as datas em que ocorreram as intervenções de PEP e PEPRO de milho em Mato Grosso.

A partir de dados disponibilizados pelo Instituto Mato-Grossense de Economia Agropecuária (IMEA), obtiveram-se séries diárias de preços do milho de principais municípios do estado de Mato Grosso. Obtiveram-se, então, observações semanais nas amostras das séries de preços de milho de 11 municípios, da seguinte forma como mostra o Quadro 2. Foram utilizadas somente as quartas-feiras de cada semana, portanto foram trabalhados dados semanais. A escolha por trabalhar dados semanais se deveu à ocorrência de datas em que os preços não foram coletados por motivos diversos (feriados, por exemplo).

Quadro 2 - Amostras da pesquisa disponibilizadas pelo IMEA

\begin{tabular}{|c|c|c|}
\hline Município & Observações* & Início da série** \\
\hline Lucas do Rio Verde & 469 & $09 / 01 / 2008$ \\
\hline Campo Verde & 469 & $09 / 01 / 2008$ \\
\hline Rondonópolis & 469 & $09 / 01 / 2008$ \\
\hline Sapezal & 469 & $09 / 01 / 2008$ \\
\hline Sorriso & 469 & $09 / 01 / 2008$ \\
\hline Tangará da Serra & 452 & $07 / 05 / 2008$ \\
\hline Canarana & 445 & $25 / 06 / 2008$ \\
\hline Campo Novo & 445 & $25 / 06 / 2008$ \\
\hline Diamantino & 417 & $07 / 01 / 2009$ \\
\hline Nova Mutum & 417 & $07 / 01 / 2009$ \\
\hline Primavera do Leste & 417 & $07 / 01 / 2009$ \\
\hline
\end{tabular}

$(*)$ Semanal $\quad(* *)$ Todos os dados referem-se às quartas-feiras.

Fonte: Elaboração própria.

A outra fonte de dados foram as informações de AGF, PEP e PEPRO disponibilizadas via e-mail pela Gerência de Formação e Controle de Estoques da Companhia Nacional de Abastecimento (CONAB). Tais dados se referem às datas em que foram divulgados pela Conab os avisos de AGF, PEP ou PEPRO. A presente pesquisa é levada a acreditar que, pelo próprio trabalho que a CONAB e os demais representantes do agronegócio brasileiro vêm desempenhando, os avisos de PGPM têm amplo conhecimento dos agentes econômicos envolvidos nas transações de milho em Mato Grosso. 
Como consta nos avisos da CONAB, após sua divulgação, os produtores têm um determinado período (cerca de três meses) para apresentar documentos fiscais comprovando a participação na política (CONAB, 2005). No entanto, pela falta de literatura que aponte outra data mais adequada, a presente pesquisa trabalhará com a data em que o aviso foi divulgado.

Tanto os dados de PEP quanto de PEPRO foram disponibilizados por sub-regiões de Mato Grosso. No entanto o AGF foi acionado para todo o estado, onde uma sub-região pode interferir nos efeitos causados em outra sub-região. Sendo assim, optou-se por não avaliar o instrumento AGF no contexto desta pesquisa. Além disso, um aviso de PEP referente ao dia 14/11/2008 não possui localidade específica, sendo destinado a todo estado de Mato Grosso, assim como ocorrido com o AGF. Por esse motivo, esse aviso em específico também será desconsiderado na análise.

Procedendo dessa maneira, o presente trabalho busca captar de forma mais isolada os efeitos da PGPM sobre os preços agrícolas. As sub-regiões de Mato Grosso foram segmentadas da seguinte forma, como mostra o Quadro 3.

Quadro 3 - Sub-regiões da Conab para os municípios analisados

\begin{tabular}{|c|c|c|c|}
\hline $\begin{array}{c}\text { Centro Norte/ } \\
\text { MT-2 }\end{array}$ & $\begin{array}{c}\text { Centro Sul/ } \\
\text { MT-3 }\end{array}$ & $\begin{array}{c}\text { Nordeste/ } \\
\text { MT-4 }\end{array}$ & $\begin{array}{c}\text { Sudeste/ } \\
\text { MT-5 }\end{array}$ \\
\hline L. do Rio Verde & Sapezal & \multirow{2}{*}{ Canarana } & Campo Verde \\
\hline Sorriso & Tangará da Serra & Rondonópolis \\
\hline \multirow{2}{*}{ Nova Mutum } & Campo Novo & & Primavera do Leste \\
\cline { 2 - 2 } & Diamantino & & \\
\cline { 2 - 2 } & & &
\end{tabular}

Fonte: Elaboração própria.

Assim, trabalhou-se com a média dos preços nominais dos municípios integrantes de cada sub-região. Desta forma, quatro séries de preços foram analisadas: MT2, MT3, MT4 e MT5, referentes às médias dos preços nominais dos municípios que compõem cada região. Para apresentação gráfica, essas séries foram deflacionadas pelo Índice Geral de Preços Disponibilidade Interna (IGP-DI) da plataforma FGV-DADOS, com base em dezembro de 2016.

As amostras analisadas continham 417 observações (para calcular a média, ajustaramse as amostras dos municípios ao tamanho do menor), exceto MT-4, composta apenas pelo município de Canarana, com 445 observações. A estrutura do modelo de intervenção dá-se da seguinte forma:

$$
y_{t}=\sum_{1}^{p} \alpha_{p} Y_{t-p}+\sum_{1}^{q} \beta_{q} \varepsilon_{t-q}+\sum_{1}^{w} \omega_{w} I N T_{w}+\varepsilon_{t}
$$

Assim, $\omega_{w}$ são os parâmetros de INT (intervenção) tipo pulse inseridos no modelo em formato de variáveis binárias, assumindo valor 1 para o período referente à data em que a Conab divulgou os avisos (ou no disponível subsequente) de PEP e PEPRO e 0 para os demais períodos da amostra. Se algum coeficiente $\omega_{w}$ for significativo e positivo (negativo), significa que a intervenção em questão aumentou (reduziu) os preços do milho para a região analisada. A variável independente $Y_{t}$ é a própria série analisada, $\alpha_{p}$ são os parâmetros autorregressivos e $\beta_{q}$ são os parâmetros de média móvel dos modelos da classe ARIMA. Segundo Souza (1991), os 
efeitos causados por características de tendência, sazonalidade e erro aleatório podem influenciar o nível ou a direção da série, por isso, utiliza-se a metodologia Box e Jenkins (1976), pois esses três elementos são tratados quando o componente residual é modelado por um ARIMA.

O primeiro passo para se estimar um modelo ARIMA é a identificação de estacionariedade da série, por meio dos testes de raiz unitária. Foram feitos três testes de raiz unitária: (1) Teste Dickey-Fuller Aumentado (ADF); (2) Teste Dickey-Fuller GLS (ADF-GLS) e; (3) Teste KwiatkowskiPhillips-Schmidt-Shin (KPSS). Caso as séries apresentem raiz unitária, procede-se a diferenciação para torná-las estacionárias. Normalmente, uma diferenciação já é suficiente.

O número de componentes autorregressivos e de médias móveis, $p$ e $q$, respectivamente, podem ser identificados a partir das Funções de Autorrelação (FAC) e Autocorreção parcial (FACP) estimadas. Morettin e Toloi (2006) sugerem que, quando as formas da FAC e da FACP são complicadas, não ficando evidente a quantidade teórica específica, deve-se estimar alguns modelos ARIMA de ordem baixa e utilizar critérios que indiquem o modelo mais adequado. 0 critério de seleção do modelo escolhido foi o AIC (Akaike's Information Criterion).

Ao se estimar o modelo, os módulos da raiz inversa dos parâmetros AR devem estar dentro do círculo unitário para garantir estacionariedade e os módulos de raiz inversa dos parâmetros MA devem permanecer dentro do círculo unitário para garantir inversibilidade. Estacionariedade e inversibilidade são pressupostos que garantem a estabilidade dos modelos ARIMA. Ainda, segundo a metodologia Box-Jenkins, é necessário que o modelo apresente resíduos ruído branco, sendo utilizada a análise do correlograma dos resíduos como indicador de resíduos ruído branco. Outro teste sobre os resíduos é o teste de heterocedasticidade de White, pois a presença de heterocedasticidade compromete os erros-padrão dos coeficientes estimados.

Em resumo, serão tomados os seguintes passos: (1) testes de estacionariedade sobre a série que será analisada; (2) ordenamento crescente dos modelos ARIMA para identificar os que têm o menor critério de seleção AIC; (3) estimação do modelo ARIMA e análise do correlograma dos resíduos - caso os resíduos sejam não correlacionados, segue-se; (4) testes de inversibilidade e estacionariedade do modelo, por meio dos testes de raízes inversas - caso o modelo seja inversível e estacionário, segue-se; (5) teste de heterocedasticidade de White - caso não seja detectada presença de heterocedasticidade nos resíduos, segue-se; (6) quantidade de parâmetros AR e MA significativos - caso os parâmetros sejam significativos, então o modelo é considerado adequado.

Caso o modelo ARIMA não passe em algum dos testes, o próximo modelo com menor critério de seleção é então submetido ao mesmo procedimento, até se encontrar um modelo que melhor se ajuste aos dados. Ao final, o modelo foi então submetido à análise de intervenção com as variáveis binárias, que foram inseridas como regressores.

\section{RESULTADOS E DISCUSSÃO}

As séries reais dos preços de milho nas sub-regiões MT2, MT3, MT4 e MT5 são apresentadas graficamente na Figura 1. 
Figura 1 - Séries MT2, MT3, MT4 e MT5 de preço do milho*

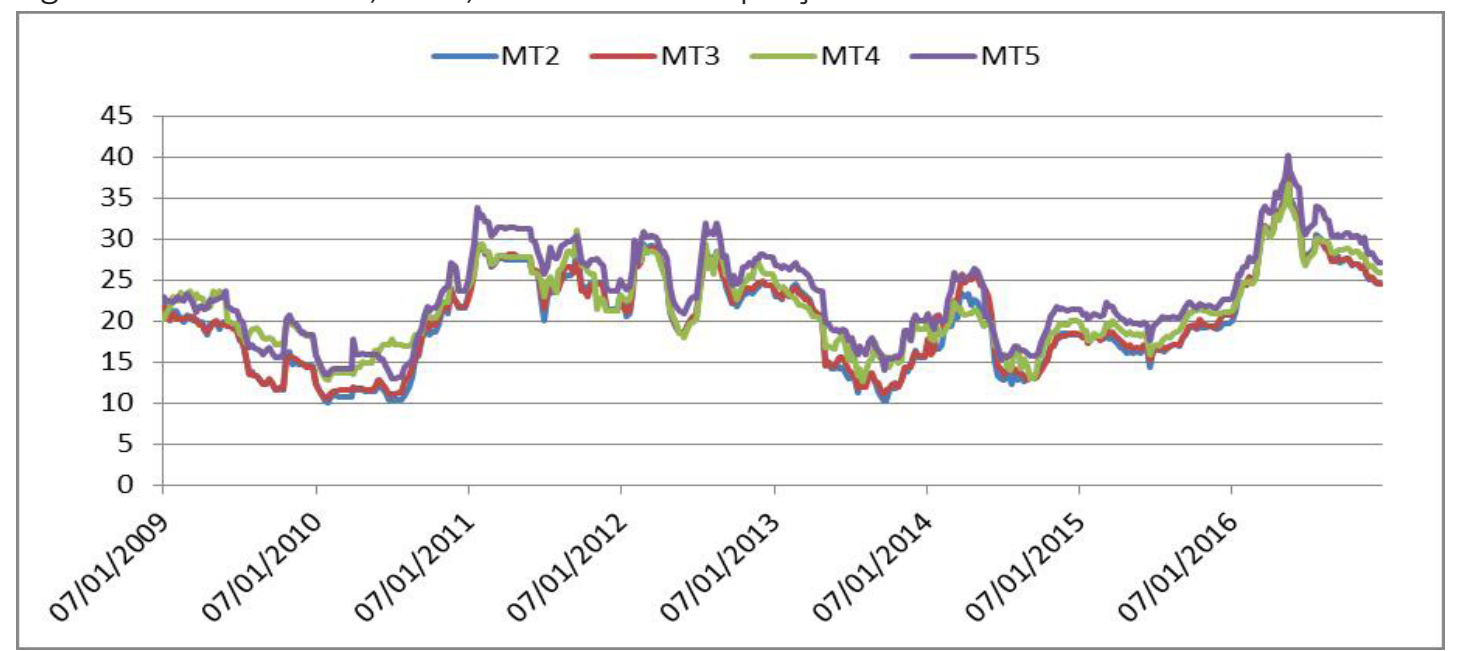

(*) Deflacionado com base em dezembro de 2016.

Fonte: Elaboração própria a partir de dados do IMEA.

Os testes de estacionariedade para as séries MT2, MT3, MT4 e MT5 indicaram a presença de raiz unitária em todas as séries em nível com tendência e constante significativas. Em primeira diferença, as séries se tornaram estacionárias sem constante e sem tendência. O que se pode observar é que uma parte dos coeficientes das intervenções não se comportou da forma como esperado, ou seja, foram não significativos ou negativos. Os resultados dos modelos estão apresentados nos Quadros 4, 5, 6 e 7.

Quadro 4 - ARIMA $(2,1,1)$ para série MT2 com dummies de intervenção

\begin{tabular}{|c|c|c|c|}
\hline Data* & Variável & Coeficiente & Prob. \\
\hline $\mathbf{2 8 / 0 7 / 2 0 0 9}$ & MT2_PEP1 & $-\mathbf{0 . 9 9}$ & $\mathbf{0 . 0 0 0}$ \\
\hline $\mathbf{2 8 / 0 8 / 2 0 0 9}$ & MT2_PEP2 & $-\mathbf{0 . 3 0}$ & $\mathbf{0 . 0 0 0}$ \\
\hline $\mathbf{0 4 / 0 9 / 2 0 0 9}$ & MT2_PEP3 & $\mathbf{0 . 2 2}$ & $\mathbf{0 . 0 0 0}$ \\
\hline $\mathbf{1 5 / 0 9 / 2 0 0 9}$ & MT2_PEP4 & $\mathbf{0 . 2 8}$ & $\mathbf{0 . 0 0 1}$ \\
\hline $\mathbf{2 4 / 0 9 / 2 0 0 9}$ & MT2_PEP5 & $\mathbf{- 0 . 3 6}$ & $\mathbf{0 . 0 0 0}$ \\
\hline $\mathbf{2 0 / 1 0 / 2 0 0 9}$ & MT2_PEP6 & $-\mathbf{0 . 1 7}$ & $\mathbf{0 . 0 0 1}$ \\
\hline $\mathbf{2 7 / 1 0 / 2 0 0 9}$ & MT2_PEP7 & $\mathbf{2 . 7 7}$ & $\mathbf{0 . 0 0 0}$ \\
\hline $\mathbf{0 5 / 1 1 / 2 0 0 9}$ & MT2_PEP8 & $\mathbf{- 0 . 8 9}$ & $\mathbf{0 . 0 0 0}$ \\
\hline $27 / 05 / 2010$ & MT2_PEP9 & -0.12 & 0.492 \\
\hline $\mathbf{0 8 / 0 6 / 2 0 1 0}$ & MT2_PEP10 & $-\mathbf{0 . 3 4}$ & $\mathbf{0 . 0 0 0}$ \\
\hline 17/06/2010 & MT2_PEP11 & -0.17 & 0.133 \\
\hline $01 / 07 / 2010$ & MT2_PEP12 & 0.08 & 0.210 \\
\hline $08 / 07 / 2010$ & MT2_PEP13 & 0.10 & 0.130 \\
\hline 15/07/2010 & MT2_PEP14 & 0.01 & 0.838 \\
\hline 22/07/2010 & MT2_PEP15 & 0.07 & 0.364 \\
\hline $\mathbf{2 9 / 0 7 / 2 0 1 0}$ & MT2_PEP16 & $\mathbf{0 . 2 8}$ & $\mathbf{0 . 0 0 8}$ \\
\hline $\mathbf{0 5 / 0 8 / 2 0 1 0}$ & MT2_PEP17 & $\mathbf{0 . 3 0}$ & $\mathbf{0 . 0 0 0}$ \\
\hline $12 / 08 / 2010$ & MT2_PEP18 & 0.26 & 0.344 \\
\hline $\mathbf{1 5 / 0 9 / 2 0 1 0}$ & MT2_PEP19 & $\mathbf{1 . 0 0}$ & $\mathbf{0 . 0 0 0}$ \\
\hline $\mathbf{2 1 / 0 7 / 2 0 0 9}$ & MT2_PEPRO1 & $\mathbf{- 1 . 0 0}$ & $\mathbf{0 . 0 0 0}$ \\
\hline $\mathbf{0 4 / 0 8 / 2 0 0 9}$ & MT2_PEPRO2 & $\mathbf{0 . 1 1}$ & $\mathbf{0 . 0 7 7}$ \\
\hline
\end{tabular}




\begin{tabular}{|c|c|c|c|}
\hline Data* & Variável & Coeficiente & Prob. \\
\hline $\mathbf{1 0 / 1 2 / 2 0 0 9}$ & MT2_PEPRO3E4 & $-\mathbf{0 . 3 2}$ & $\mathbf{0 . 0 0 5}$ \\
\hline 19/08/2010 & MT2_PEPRO5 & 0.53 & 0.126 \\
\hline $\mathbf{1 6 / 0 7 / 2 0 1 3}$ & MT2_PEPRO6 & $-\mathbf{0 . 3 4}$ & $\mathbf{0 . 0 4 4}$ \\
\hline $\mathbf{2 4 / 0 7 / 2 0 1 3}$ & MT2_PEPRO7 & $\mathbf{- 1 . 4 3}$ & $\mathbf{0 . 0 0 0}$ \\
\hline $\mathbf{1 3 / 0 8 / 2 0 1 3}$ & MT2_PEPRO8 & $\mathbf{0 . 6 4}$ & $\mathbf{0 . 0 0 0}$ \\
\hline $\mathbf{2 0 / 0 8 / 2 0 1 3}$ & MT2_PEPRO9 & $\mathbf{0 . 8 3}$ & $\mathbf{0 . 0 0 0}$ \\
\hline $\mathbf{2 7 / 0 8 / 2 0 1 3}$ & MT2_PEPRO10 & $\mathbf{- 0 . 4 3}$ & $\mathbf{0 . 0 0 2}$ \\
\hline $\mathbf{2 0 / 0 9 / 2 0 1 3}$ & MT2_PEPRO11 & $-\mathbf{0 . 4 1}$ & $\mathbf{0 . 0 2 9}$ \\
\hline $01 / 10 / 2013$ & MT2_PEPRO12 & -0.08 & 0.786 \\
\hline $25 / 10 / 2013$ & MT2_PEPRO13 & 0.05 & 0.838 \\
\hline $\mathbf{1 2 / 1 1 / 2 0 1 3}$ & MT2_PEPRO14 & $\mathbf{0 . 5 3}$ & $\mathbf{0 . 0 0 4}$ \\
\hline $\mathbf{2 2 / 1 1 / 2 0 1 3}$ & MT2_PEPRO15 & $\mathbf{0 . 7 3}$ & $\mathbf{0 . 0 1 1}$ \\
\hline $\mathbf{2 0 / 0 8 / 2 0 1 4}$ & MT2_PEPRO16 & $\mathbf{0 . 7 3}$ & $\mathbf{0 . 0 0 0}$ \\
\hline $28 / 08 / 2014$ & MT2_PEPRO17 & 0.21 & 0.159 \\
\hline $\mathbf{1 1 / 0 9 / 2 0 1 4}$ & MT2_PEPRO18 & $-\mathbf{0 . 4 7}$ & $\mathbf{0 . 0 0 1}$ \\
\hline $18 / 09 / 2014$ & MT2_PEPRO19 & 0.02 & 0.907 \\
\hline- & $\boldsymbol{\alpha}_{\mathbf{1}}$ & $\mathbf{- 0 . 5 5}$ & $\mathbf{0 . 0 0 5}$ \\
\hline- & $\boldsymbol{\alpha}_{2}$ & $\mathbf{0 . 2 2}$ & $\mathbf{0 . 0 0 3}$ \\
\hline- & $\boldsymbol{\beta}_{\mathbf{1}}$ & $\mathbf{0 . 7 1}$ & $\mathbf{0 . 0 0 0}$ \\
\hline
\end{tabular}

$\left(^{*}\right)$ Data referente ao aviso da Conab.

Fonte: Elaboração própria.

Quadro 5 - ARIMA $(5,1,4)$ para série MT3 com dummies de intervenção

\begin{tabular}{|c|c|c|c|}
\hline Data* & Variável & Coeficiente & Prob. \\
\hline $\mathbf{2 7 / 0 5 / 2 0 1 0}$ & MT3_PEP1 & $\mathbf{0 . 6 9}$ & $\mathbf{0 . 0 0 0}$ \\
\hline $08 / 06 / 2010$ & MT3_PEP2 & -0.08 & 0.729 \\
\hline $17 / 06 / 2010$ & MT3_PEP3 & -0.14 & 0.354 \\
\hline $01 / 07 / 2010$ & MT3_PEP4 & 0.15 & 0.459 \\
\hline $08 / 07 / 2010$ & MT3_PEP5 & -0.08 & 0.657 \\
\hline $15 / 07 / 2010$ & MT3_PEP6 & 0.18 & 0.575 \\
\hline $22 / 07 / 2010$ & MT3_PEP7 & -0.06 & 0.788 \\
\hline $\mathbf{2 9 / 0 7 / 2 0 1 0}$ & MT3_PEP8 & $\mathbf{1 . 0 4}$ & $\mathbf{0 . 0 0 0}$ \\
\hline $05 / 08 / 2010$ & MT3_PEP9 & 0.03 & 0.920 \\
\hline $12 / 08 / 2010$ & MT3_PEP10_PEPRO1 & 0.18 & 0.425 \\
\hline $\mathbf{1 5 / 0 9 / 2 0 1 0}$ & MT3_PEP11 & $\mathbf{0 . 8 0}$ & $\mathbf{0 . 0 1 9}$ \\
\hline $16 / 07 / 2013$ & MT3_PEPRO2 & -0.28 & 0.290 \\
\hline $\mathbf{2 4 / 0 7 / 2 0 1 3}$ & MT3_PEPRO3 & $\mathbf{- 1 . 8 8}$ & $\mathbf{0 . 0 0 0}$ \\
\hline 13/08/2013 & MT3_PEPRO4 & 0.17 & 0.621 \\
\hline $\mathbf{2 0 / 0 8 / 2 0 1 3}$ & MT3_PEPRO5 & $\mathbf{1 . 2 1}$ & $\mathbf{0 . 0 0 0}$ \\
\hline $27 / 08 / 2013$ & MT3_PEPRO6 & -0.58 & 0.114 \\
\hline $\mathbf{2 0 / 0 9 / 2 0 1 3}$ & MT3_PEPRO7 & $-\mathbf{0 . 8 9}$ & $\mathbf{0 . 0 1 5}$ \\
\hline $01 / 10 / 2013$ & MT3_PEPRO8 & 0.23 & 0.625 \\
\hline $\mathbf{2 5 / 1 0 / 2 0 1 3}$ & MT3_PEPRO9 & $\mathbf{- 1 . 0 8}$ & $\mathbf{0 . 0 1 9}$ \\
\hline $12 / 11 / 2013$ & MT3_PEPRO10 & 0.64 & 0.111 \\
\hline $22 / 11 / 2013$ & MT3_PEPRO11 & -0.43 & 0.309 \\
\hline $20 / 08 / 2014$ & MT3_PEPRO12 & 0.37 & 0.286 \\
\hline $28 / 08 / 2014$ & MT3_PEPRO13 & -0.27 & 0.397 \\
\hline
\end{tabular}




\begin{tabular}{|c|c|c|c|}
\hline $11 / 09 / 2014$ & MT3_PEPRO14 & -0.06 & 0.885 \\
\hline $18 / 09 / 2014$ & MT3_PEPRO15 & 0.19 & 0.540 \\
\hline- & $\boldsymbol{\alpha}_{1}$ & $\mathbf{- 1 . 0 5}$ & $\mathbf{0 . 0 0 0}$ \\
\hline- & $\boldsymbol{\alpha}_{2}$ & $-\mathbf{0 . 3 8}$ & $\mathbf{0 . 0 0 0}$ \\
\hline- & $\boldsymbol{\alpha}_{3}$ & $-\mathbf{0 . 8 4}$ & $\mathbf{0 . 0 0 0}$ \\
\hline- & $\boldsymbol{\alpha}_{4}$ & $\mathbf{- 0 . 4 3}$ & $\mathbf{0 . 0 0 0}$ \\
\hline- & $\boldsymbol{\alpha}_{5}$ & $\mathbf{0 . 3 0}$ & $\mathbf{0 . 0 0 0}$ \\
\hline- & $\boldsymbol{\beta}_{1}$ & $\mathbf{1 . 2 7}$ & $\mathbf{0 . 0 0 0}$ \\
\hline- & $\boldsymbol{\beta}_{2}$ & $\mathbf{0 . 7 6}$ & $\mathbf{0 . 0 0 0}$ \\
\hline- & $\boldsymbol{\beta}_{3}$ & $\mathbf{1 . 2 6}$ & $\mathbf{0 . 0 0 0}$ \\
\hline- & $\boldsymbol{\beta}_{4}$ & $\mathbf{0 . 9 8}$ & $\mathbf{0 . 0 0 0}$ \\
\hline
\end{tabular}

$\left.{ }^{*}\right)$ Data referente ao aviso da Conab.

Fonte: Elaboração própria.

Quadro 6-ARIMA $(6,1,4)$ para série MT4 com dummies de intervenção

\begin{tabular}{|c|c|c|c|}
\hline Data & Variável & Coeficiente & Prob. \\
\hline $27 / 05 / 2010$ & MT4_PEP1 & 0.51 & 0.306 \\
\hline $08 / 06 / 2010$ & MT4_PEP2 & -0.26 & 0.230 \\
\hline $17 / 06 / 2010$ & MT4_PEP3 & -0.74 & 0.362 \\
\hline $01 / 07 / 2010$ & MT4_PEP4 & 1.01 & 0.134 \\
\hline $08 / 07 / 2010$ & MT4_PEP5 & -0.67 & 0.005 \\
\hline $15 / 07 / 2010$ & MT4_PEP6 & 0.15 & 0.730 \\
\hline $22 / 07 / 2010$ & MT4_PEP7 & -0.77 & 0.428 \\
\hline $29 / 07 / 2010$ & MT4_PEP8 & 0.21 & 0.606 \\
\hline $05 / 08 / 2010$ & MT4_PEP9 & 0.64 & 0.405 \\
\hline $12 / 08 / 2010$ & MT4_PEP10 & -0.22 & 0.544 \\
\hline $15 / 09 / 2010$ & MT4_PEP11 & 0.84 & 0.254 \\
\hline $19 / 08 / 2010$ & MT4_PEPRO1 & 0.39 & 0.422 \\
\hline $13 / 08 / 2013$ & MT4_PEPRO2 & 1.52 & 0.001 \\
\hline $20 / 08 / 2013$ & MT4_PEPRO3 & 0.82 & 0.065 \\
\hline $27 / 08 / 2013$ & MT4_PEPRO4 & 0.53 & 0.293 \\
\hline $25 / 10 / 2013$ & MT4_PEPRO5 & -1.15 & 0.022 \\
\hline $22 / 11 / 2013$ & MT4_PEPRO6 & -0.71 & 0.039 \\
\hline $20 / 08 / 2014$ & MT4_PEPRO7 & -2.50 & 0.000 \\
\hline $28 / 08 / 2014$ & MT4_PEPRO8 & -1.05 & 0.016 \\
\hline $11 / 09 / 2014$ & MT4_PEPRO9 & -0.25 & 0.717 \\
\hline $18 / 09 / 2014$ & MT4_PEPRO10 & 0.91 & 0.065 \\
\hline- & $\alpha_{1}$ & -0.95 & 0.000 \\
\hline- & $\alpha_{2}$ & -0.74 & 0.000 \\
\hline- & $\alpha_{3}$ & -0.74 & 0.000 \\
\hline- & $\alpha_{4}$ & -0.64 & 0.000 \\
\hline- & $\alpha_{5}$ & 0.19 & 0.042 \\
\hline- & $\alpha_{6}$ & 0.16 & 0.013 \\
\hline- & $\beta_{1}$ & 1.02 & 0.000 \\
\hline- & $\beta_{2}$ & 0.92 & 0.000 \\
\hline- & $\beta_{3}$ & 1.01 & 0.000 \\
\hline- & $\beta_{4}$ & 0.99 & 0.000 \\
\hline
\end{tabular}

(*) Data referente ao aviso da Conab.

Fonte: Elaboração própria. 
Quadro 7 - ARIMA $(2,1,4)$ para série MT5 com dummies de intervenção

\begin{tabular}{|c|c|c|c|}
\hline Data* & Variável & Coeficiente & Prob. \\
\hline $27 / 05 / 2010$ & MT5_PEP1 & -0.09 & 0.393 \\
\hline $\mathbf{0 8 / 0 6 / 2 0 1 0}$ & MT5_PEP2 & $\mathbf{- 0 . 2 3}$ & $\mathbf{0 . 0 4 5}$ \\
\hline $\mathbf{1 7 / 0 6 / 2 0 1 0}$ & MT5_PEP3 & $-\mathbf{0 . 4 3}$ & $\mathbf{0 . 0 0 0}$ \\
\hline $\mathbf{0 1 / 0 7 / 2 0 1 0}$ & MT5_PEP4 & $\mathbf{- 0 . 5 1}$ & $\mathbf{0 . 0 0 0}$ \\
\hline $08 / 07 / 2010$ & MT5_PEP5 & 0.09 & 0.374 \\
\hline $\mathbf{1 5 / 0 7 / 2 0 1 0}$ & MT5_PEP6 & $\mathbf{0 . 2 3}$ & $\mathbf{0 . 0 8 0}$ \\
\hline $22 / 07 / 2010$ & MT5_PEP7 & -0.03 & 0.869 \\
\hline $\mathbf{2 9 / 0 7 / 2 0 1 0}$ & MT5_PEP8 & $\mathbf{0 . 8 2}$ & $\mathbf{0 . 0 0 0}$ \\
\hline $05 / 08 / 2010$ & MT5_PEP9 & 0.15 & 0.499 \\
\hline $12 / 08 / 2010$ & MT5_PEP10 & 0.26 & 0.126 \\
\hline $20 / 08 / 2014$ & MT5_PEPRO1_PEPRO2 & 0.01 & 0.982 \\
\hline $\mathbf{2 8 / 0 8 / 2 0 1 4}$ & MT5_PEPRO3 & $\mathbf{1 . 0 0}$ & $\mathbf{0 . 0 2 5}$ \\
\hline- & $\boldsymbol{\alpha}_{\mathbf{1}}$ & $\mathbf{0 . 4 7}$ & $\mathbf{0 . 0 0 1}$ \\
\hline- & $\boldsymbol{\alpha}_{2}$ & $\mathbf{- 0 . 7 5}$ & $\mathbf{0 . 0 0 0}$ \\
\hline- & $\boldsymbol{\beta}_{\mathbf{1}}$ & $\mathbf{- 0 . 3 3}$ & $\mathbf{0 . 0 2 8}$ \\
\hline- & $\boldsymbol{\beta}_{\boldsymbol{2}}$ & $\mathbf{0 . 8 4}$ & $\mathbf{0 . 0 0 0}$ \\
\hline- & $\boldsymbol{\beta}_{\mathbf{3}}$ & $\mathbf{0 . 1 7}$ & $\mathbf{0 . 0 0 4}$ \\
\hline- & $\boldsymbol{\beta}_{\mathbf{4}}$ & $\mathbf{0 . 1 5}$ & $\mathbf{0 . 0 7 5}$ \\
\hline
\end{tabular}

$\left.{ }^{*}\right)$ Data referente ao aviso da Conab.

Fonte: Elaboração própria.

O Quadro 8 sintetiza os resultados dos modelos apresentando o modelo estimado, a quantidade de parâmetros significativos e positivos e de cada tipo de intervenção (PEP ou PEPRO) para cada sub-região.

Quadro 8 - Síntese dos Resultados

\begin{tabular}{|c|c|c|c|c|c|}
\hline Série & Modelo & $\begin{array}{c}\text { PEP } \\
\text { Significativo }\end{array}$ & $\begin{array}{c}\text { PEP } \\
\text { Positivo* }\end{array}$ & $\begin{array}{c}\text { PEPRO } \\
\text { Significativo }\end{array}$ & $\begin{array}{c}\text { PEPRO } \\
\text { Positivo* }\end{array}$ \\
\hline MT2 & ARIMA $(2,1,1)$ & $12 / 19$ & $6 / 12$ & $13 / 18$ & $6 / 13$ \\
\hline MT3 & ARIMA $(5,1,4)$ & $3 / 11$ & $3 / 3$ & $4 / 15$ & $1 / 4$ \\
\hline MT4 & ARIMA $(6,1,4)$ & $1 / 11$ & $0 / 1$ & $7 / 10$ & $3 / 7$ \\
\hline MT5 & ARIMA $(2,1,4)$ & $5 / 10$ & $2 / 5$ & $1 / 3$ & $1 / 1$ \\
\hline
\end{tabular}

(*) Positivos e significativos.

Fonte: Elaboração própria.

Os resultados apresentados para as quatro sub-regiões de Mato Grosso vão de encontro às afirmações de Sanches e Bacha (2015). Os autores analisaram os preços do mercado de milho no estado de Mato Grosso no período de 2009 a 2014 e concluíram que os instrumentos de PEP e PEPRO (também analisaram conjuntamente os Contratos de Opção de Venda de Produtos Agrícolas) "têm sido eficazes em elevar os preços de mercado". No entanto, pelos resultados encontrados, não é possível fazer tal afirmação de eficácia para o PEP e PEPRO de milho em Mato Grosso, no período de 2009 a 2016.

Saraiva et al. (2014), ao realizarem análise de intervenção de Contrato de Opção de Venda (COV) e PEP sobre os preços do arroz no Rio Grande do Sul, perceberam que apenas três das 
14 intervenções via esses instrumentos de PGPM foram significativas. Os autores perceberam que essa significância nos parâmetros ocorreu em momentos que foram aportados valores maiores pelo Governo Federal à política, que coincidiu em momentos de crise da orizicultura. No entanto o presente trabalho não buscou avaliar se os resultados encontrados foram causados pela quantidade de recursos destinada às intervenções via PEP e PEPRO de milho em Mato Grosso. De qualquer modo, é possível afirmar que, pelas evidências encontradas em Schwantes e Bacha (2017), haveria um ônus social maior caso os recursos tivessem sido redirecionados aos instrumentos da Política de Compra de Excedentes pelo Governo (PCEG) e para as Compras Diretas da Agricultura Familiar (CDAF). Ainda, a partir dos resultados de Schwantes e Bacha (2017), é possível afirmar que o custo social da intervenção via PGPM teria sido menor caso os recursos tivessem sido redirecionados à Política de Seguro de Preços (PPS).

\section{CONSIDERAÇÕES FINAIS}

De modo geral, os modelos ARIMA se ajustaram bem às séries de MT2, MT3, MT4 e MT5, o que possibilitou o cumprimento do objetivo geral deste trabalho, que foi estimar modelos de intervenção de PEP e PEPRO sobre essas séries. No entanto a hipótese que norteava a pesquisa não foi confirmada. Inicialmente, acreditava-se que a divulgação das operações de PEP e PEPRO pela Conab geraria efeitos positivos e significativos sobre as séries de preços do milho em Mato Grosso. Porém, a partir dos resultados encontrados, não foi possível confirmar tal hipótese de maneira geral, pois, para a maioria das intervenções, foram encontrados coeficientes não significativos e também muitos coeficientes negativos e significativos.

Duas outras hipóteses surgem desses resultados, outros fatores além dos instrumentos de PGPM (PEP e PEPRO, neste caso) exercem pressão sobre os preços de milho em Mato Grosso, enquanto esses estão em níveis baixos (próximos ou abaixo do preço mínimo). Ou então a data escolhida para inserir as variáveis binárias de intervenção não foram as adequadas, sendo possível que esse efeito ocorra com uma defasagem em relação à data de divulgação da política pela Conab (não analisada pelo presente trabalho). De qualquer modo, os resultados aqui apresentados podem contribuir para esta e outras investigações.

As limitações dos resultados encontrados surgem da alta sensibilidade que os coeficientes dos modelos ARIMA apresentam, pequenas alterações realizadas no processo de modelagem podem gerar alterações importantes no contexto de se analisar os valores e os sinais dos coeficientes. No entanto esse tipo de modelagem, inclusive pelos testes realizados, permite confiar nos testes de significância. Outra limitação surge do fato de se ter trabalhado com dados semanais; como consequência, não se modelou a sazonalidade, por ela não estar presente nos testes realizados.

Como implicação de políticas públicas, nota-se que, caso validados pela comunidade os resultados aqui encontrados, o custo de oportunidade dos recursos destinados ao PEP e PEPRO do milho em Mato Grosso aumentam. Assim, podem ser pensadas estratégias alternativas na busca de garantia de renda mínima aos produtores de milho desse estado, como fomentar o seguro rural. 


\section{REFERÊNCIAS}

ABREU, D. P.; WANDER, A. E.; FERREIRA, M. D. P. In: CONGRESSO SOCIEDADE BRASILEIRA DE ECONOMIA, ADMINISTRAÇÃO E SOCIOLOGIA RURAL, 55., 2017, Santa Maria, RS. Análise de artigos científicos sobre a política de garantira de preços mínimos no Brasil: publicados a partir de 2000. Anais [...]. Santa Maria, 2017.

BACHA, C. J. C.; CALDARELLI, C. E. Avaliação do desempenho dos novos instrumentos de Política de Garantia de Preços Agrícola de 2004 a 2007. In: CONGRESSO DA SOCIEDADE BRASILEIRA DE ECONOMIA, ADMINISTRAÇÃO E SOCIOLOGIA RURAL, 46., 2008, Rio Branco, AC. Anais [...]. Rio Branco: SOBER, 2008. [CD-ROM].

BITENCOURT, M. B.; BARCZSZ, S. S.; ANDRADE, A. A. Análise da PGPM no mercado brasileiro de algodão em pluma, milho e trigo, utilizando como instrumento de intervenção a AGF, Contrato de Opção, PEP e PEPRO. In: CONGRESSO DA SOCIEDADE BRASILEIRA DE ECONOMIA, ADMINISTRAÇÃO E SOCIOLOGIA RURAL, 46., 2008, Rio Branco, AC. Anais [...]. Rio Branco: SOBER, 2008. [CD-ROM].

BORGATTO, A. F.; SÁFADI, T. Análise de Intervenção em séries temporais: aplicações em transporte urbano. Revista Brasileira de Estatística, Rio de Janeiro, v. 61, n. 215, p. 81-102, 2000.

BORGES, A. H.; PINHO, J.; AZEVEDO, J. P.; NEWMAN, J.; JOPPERT, M. P.; CARVALHO, S. N. A rede brasileira de monitoramento e avaliação: um relato do processo de sua criação e desenvolvimento. Revista Brasileira de Monitoramento e Avaliação, n. 1, jan./jun. 2011.

BOX, G. E. P.; JENKINS, G. M. Time series analysis forecasting and control. [Edição revisada]. San Francisco: Holden Day, 1976.

BOX, G. E. P.; TIAO, G. C. Intervention with Applications Analysis to Economic and Environmental Problems. Journal of the American Statistical Association, v. 70, n. 349, p. 70-9, 1975.

BRASIL. Ministério de Agricultura, Pecuária e Abastecimento. Plano Agrícola e Pecuário 2016-2017. Brasília: MAPA/SPA, 2016.

CALDARELLI, C. E.; BACCHI, M. R. P. Fatores de influência no preço do milho no Brasil. Nova Economia, v. 22, n. 1, p. 141-64, 2012.

CHAIN, C. P.; BERTOLINO, A. C. N.; SIQUEIRA, L. C.; SANTOS, A. C. Intervenções na distância econômica entre Brasil e Argentina a partir da constituição do Mercosul. Perspectiva Econômica, São Leopoldo, v. 12, n. 2, p. 17-34, jul./dez. 2016.

COMPANHIA NACIONAL DE ABASTECIMENTO. Regulamento para operacionalização da oferta de prêmio de risco para aquisição de produto agropecuário oriundo de contrato privado de opção de venda PROP N. 001/2005. Brasília-DF, 2005. Disponível em: http://www.conab.gov.br/OlalaCMS/uploads/ arquivos/10_10_26_20_10_48_regulamentoprop_001_2005.pdf. Acesso em: jan. 2018.

CONCEIÇÃO, J. C. P. R. Contribuição dos novos instrumentos de comercialização (Contratos de opção e PEP) para a estabilização de preço e renda agrícolas. Texto para Discussão n. 927. Brasília: IPEA, 2002. Disponível em: http://www.ipea.gov.br/portal/index.php?option=com_content\&view=article\&id=4161. Acesso em: jan. 2017.

CRUZ, A. C.; TEIXEIRA, E. C. Desempenho da política de garantia de preços via contratos de opção de venda e prêmio para escoamento de produto. Revista de Política Agrícola, Brasília, n. 4, p. 24-36, 2006.

DEL BEL FILHO, E.; BACHA, C. J. C. Avaliação das mudanças na política de garantia de preços mínimos: período de 1997 a 2004. Revista de Economia e Agronegócio, Viçosa, MG, v. 3, n. 1, p. 51-76, 2005. 
FERREIRA, M. D. P.; SOUSA, L. O.; MATTOS, L. B.; CAMPOS, A. C. Impactos da crise financeira internacional e da influenza A sobre as exportações brasileiras de carne suína. Revista de Economia e Agronegócio, Viçosa, MG, v. 9, n. 1, p. 79-100, 2011.

HANSEN, B. T.; ØSTERGAARD, S. D.; SØNDERSKOV, K. M.; DINESEN, P. T. Increased Incidence Rate of Traumaand Stressor-Related Disorders in Denmark after the September 11, 2001, Terrorist Attacks in the United States. American Journal of Epidemiology, v. 184, n. 7, p. 494-500, 2016.

INSTITUTO BRASILEIRO DE GEOGRAFIA E ESTATÍSTICA. Produção Agrícola Municipal. 2017. Disponível em: https://sidra.ibge.gov.br/tabela/839. Acesso em: jan. 2018.

MASSUQUETTI, A.; SOUZA, O. T.; BEROLDT, L. A. Instrumentos de política agrícola e mudanças institucionais. In: CONGRESSO DA SOCIEDADE BRASILEIRA DE ECONOMIA ADMINISTRAÇÃO E SOCIOLOGIA RURAL, 48., Campo Grande, MS. 2010. Anais [...]. Campo Grande, 2010.

MORETTIN, P. A.; TOLOI, C. M. C. Análise de séries temporais. 2. ed. São Paulo: Blucher, 2006.

OSAKI, M.; BATALHA, M. O. Efeito da Política de Garantia de Preços Mínimos (PGPM) na comercialização de milho e soja nas cinco regiões geográficas do Brasil. In: CONGRESSO DA SOCIEDADE BRASILEIRA DE ECONOMIA, ADMINISTRAÇÃO E SOCIOLOGIA RURAL, 47., 2009, Porto Alegre, RS. Anais [...]. Porto Alegre: SOBER, 2009. [CD-ROM].

PEREIRA, A. C.; CARVALHO, F. M. A.; CONCEIÇÃO, J. C. P. R. Evolução e desempenho da política de contratos de opção de venda para mercados agrícolas. Revista de Economia e Agronegócio, Viçosa, MG, v. 3, n. 4, p. 487-508, 2005.

PEREIRA, M. W. G.; VIEIRA, W. C. Análise da política de garantia de preços mínimos: o caso do Prêmio para Escoamento do Produto. Revista de Política Agrícola, Brasília, n. 2, p. 6-17, 2009.

RAMOS, S. Y.; MORCELI, P. Conjuntura de Mercado e Intervenção Governamental na Agricultura: o caso da cadeia produtiva do arroz. In: CONGRESSO DA SOCIEDADE BRASILEIRA DE ECONOMIA, ADMINISTRAÇÃO E SOCIOLOGIA RURAL, 48., 2010, Campo Grande, MS. Anais [...]. Campo Grande, MS: SOBER, 2010. [CD-ROM].

RESENDE, G. M. Avaliação de Políticas Públicas no Brasil: uma análise de seus impactos regionais. 1. ed. Rio de Janeiro: Ipea, 2014a. v. 1.

RESENDE, G. M. Avaliação de Políticas Públicas no Brasil: uma análise de seus impactos regionais. Rio de Janeiro: Ipea, 2014b. v. 2.

ReSEnde, G. M. M. Avaliação de Políticas Públicas no Brasil: uma análise da Política Nacional de Desenvolvimento Regional (PNDR). 1. ed. Rio de Janeiro: Ipea, 2017. v. 3.

REZENDE, G. C. Política de preços mínimos na década de 90: Dos velhos aos novos instrumentos. Texto para Discussão n. 740. Brasília: IPEA, 2000. Disponível em: http://desafios2.ipea.gov.br/agencia/images/ stories/PDFs/TDs/td_0740.pdf. Acesso em: jan. 2018.

SANCHES, A. L. R.; BACHA, C. J. C. Políticas de estabilização de preços agrícolas: o caso do mercado do milho em Mato Grosso. In: CONGRESSO DA SOCIEDADE BRASILEIRA DE ECONOMIA, ADMINISTRAÇÃO E SOCIOLOGIA RURAL, 53., 2015, João Pessoa, PB. Anais [...]. João Pessoa: SOBER, 2015. [CD-ROM].

SARAIVA, B. S.; FERREIRA, M. D. P.; DANIEL, L. P.; CUNHA, D. A. Políticas agrícolas de garantia de preço mínimo: impactos sobre os preços do arroz no Rio Grande do Sul. Nexos Econômicos, Salvador, v. 8, n. 2, p. 67-79, 2014. 
SCHWANTES, F.; BACHA, C. J. C. Custos sociais e orçamentários das políticas de garantia de preços no Brasil - estudo dos casos de arroz e milho. Revista de Economia e Sociologia Rural, Brasília, v. 55, n. 2, p. 367-88, 2017.

SOUSA, L. O.; FERREIRA, M. D. P.; MATTOS, L. B.; CAMPOS, A. C. Análise das intervenções nas exportações de açúcar bruto do Brasil para a Rússia, de 1997 a 2010. Revista de Política Agrícola, Brasília, n. 3, nov. 2016, p. 63-76, 2010.

SOUZA, A. M. Aplicação e performance da análise de intervenção em séries macroeconômicas brasileiras. Orientadora: Maria Emília Camargo. 1991. 93f. Trabalho de Conclusão de Curso (Especialização em Estatística e Modelagem Quantitativa)- Universidade Federal de Santa Maria, Santa Maria, 1991.

WINTERS, P.; MAFFIOLI, A.; SALAZAR, L. Introduction to the special feature: evaluating the impact of agricultural projects in developing countries. Journal of Agricultural Economics, v. 62, n. 2, p. 393-402, 2011.

\section{Sobre os autores:}

Douglas Paranahyba de Abreu: Doutorando e mestre em Agronegócio pela Universidade Federal de Goiás (UFG). Esta pesquisa foi financiada com bolsa de mestrado da Fundação de Amparo à Pesquisa do Estado de Goiás (FAPEG). E-mail: abreu.douglasp@gmail.com, Orcid: http://orcid. org/0000-0002-1089-8358

Alcido Elenor Wander: Doutor em Ciências Agrárias pela University of Göttingen, Alemanha. Pesquisador da Embrapa e Professor do Programa de Pós-Graduação em Agronegócio da Universidade Federal de Goiás (UFG). E-mail: alcidowander@gmail.com, Orcid: http://orcid. org/0000-0001-9656-8773

Marcelo Dias Paes Ferreira: Doutor em Economia Aplicada pela Universidade Federal de Viçosa. Professor do Programa de Pós-Graduação em Agronegócio da Universidade Federal de Goiás (UFG). E-mail: marcelo.agronegocio@gmail.com, Orcid: http://orcid.org/0000-0001-6850-078X 
